\title{
Actualidad: Perspectiva sobre discapacidad e inclusión en educación superior
}

\author{
Actuality: A perspective on disability and inclusion in higher education \\ Atualidades: Perspectiva sobre deficiência e inclusão no ensino superior
}

\author{
Stefany Santisteban \\ scsantisteban@fucsalud.edu.co \\ https://orcid.org/0000-0003-2705-1345
}

Fundación Universitaria de Ciencias de la Salud, Bogotá-Colombia

Recibido 2 de marzo 2021 | Arbitrado y aceptado 26 de marzo 2021 | Publicado en 4 de mayo 2021

\begin{abstract}
RESUMEN
Alrededor del $15 \%$ de la población mundial se encuentra en condición de discapacidad, cifras que van en aumento debido al incremento de las enfermedades crónicas no transmisibles, el envejecimiento, la accidentalidad y la violencia, adicionalmente, se considera que algún momento de la vida todos presentaremos algún tipo de discapacidad; este concepto ha cambiado a lo largo de la historia con diversos modelos, sin embargo actualmente, bajo el marco teórico de la Clasificación Internacional del Funcionamiento, la Discapacidad y la Salud (CIF), la discapacidad es entendida no solo como alteraciones anatómicas y/o fisiológicas, sino como la limitación en la realización de las actividades y la restricción en la participación, para lo cual interactúan diversos factores contextuales. La discapacidad se relaciona con aumento en la vulnerabilidad, lo que incluye el acceso a la educación, la cual es una base para el desarrollo personal y social, asociada además con mejora en la calidad de vida y la participación de las personas en condición de discapacidad, por lo que los países e instituciones deben aunar esfuerzos, ratificar compromisos y ejecutar estrategias para favorecer la inclusión educativa.
\end{abstract}

Palabras clave: Personas con discapacidad; CIF; educación; rehabilitación
SS: Fisioterapeuta, Universidad Industrial de Santander. Magister en Neurorehabilitación, Universidad Autónoma de Manizales. Docente del programa de fisioterapia de la facultad de ciencias del movimiento de la Fundación Universitaria de Ciencias de la Salud e investigadora. Fundación Universitaria de Ciencias de la Salud, Colombia.

\begin{abstract}
Around $15 \%$ of the world population is disabled, figures that are increasing due to the increase in chronic non-communicable diseases, aging, accidents and violence, additionally, it is considered that at some point in life everyone we will present some type of disability; this concept has changed throughout history with various models, however currently, under the theoretical framework of the International Classification of Functioning, Disability and Health (ICF), disability is understood not only as anatomical alterations and/or physiological, but as the limitation in the performance of activities and the restriction in participation, for which various contextual factors interact. Disability is related to an increase in vulnerability, which includes access to education, which is a basis for personal and social development, also associated with an improvement in the quality of life and the participation of people with disabilities. Therefore, countries and institutions must join forces, ratify commitments and implement strategies to promote educational inclusion.
\end{abstract}

Key words: Disabled persons; ICF; education, rehabilitation 
SS: Fisioterapeuta, Universidad Industrial de Santander. Magister en Neurorehabilitación, Universidad Autónoma de Manizales. Docente del programa de fisioterapia de la facultad de ciencias del movimiento de la Fundación Universitaria de Ciencias de la Salud e investigadora. Fundación Universitaria de Ciencias de la Salud, Colombia.

\section{RESUMO}

Cerca de $15 \%$ da população mundial é deficiente, cifras que vêm aumentando devido ao aumento das doenças crônicas não transmissíveis, envelhecimento, acidentes e violência, além disso, considera-se que algum momento da vida é tudo que apresentaremos algum tipo de deficiência ; Este conceito mudou ao longo da história com vários modelos, porém atualmente, sob o referencial teórico da Classificação Internacional de Funcionalidade, Incapacidade e Saúde (CIF), a deficiência é entendida não apenas como alterações anatômicas e / ou fisiológicas, mas como limitação na desempenho de atividades e a restrição de participação, para as quais diversos fatores contextuais interagem. A deficiência está relacionada com o aumento da vulnerabilidade, que inclui o acesso à educação, base para o desenvolvimento pessoal e social, associada também à melhoria da qualidade de vida e à participação das pessoas com deficiência, pelo que os países e instituições devem aderir forças, ratificar compromissos e implementar estratégias para promover a inclusão educacional.

Palavras-chave: Pessoas com deficiência; CIF; educação; reabilitação

\section{PERSPECTIVAS}

A ntes de definir formalmente la palabra "discapacidad," resulta conveniente realizar una revisión histórica de los eventos que han llevado a la construcción de dicho término con el objetivo de dar una visión clara que permita no solo teorizar, sino comprender y dar el lugar que esta condición inherente a la humanidad merece en nuestra sociedad, para de este modo abordarla de manera adecuada. Es así como se han establecido tres modelos de discapacidad, que son convenciones cronológicas que no son rígidos en tiempo ni en culturas (1): el modelo de prescindencia circunscrito desde la antigüedad hasta la edad media, el modelo médico o de rehabilitación predominante hasta la mitad del siglo XX y el modelo social que surgió en la década de los 60 del siglo pasado y permanece vigente.

En el modelo de prescindencia se tenía una perspectiva mágica y religiosa de las alteraciones físicas que presentaban las personas, principalmente en los recién nacidos, por lo cual se optaba por la muerte o la marginación de estos individuos, pues se consideraba que no aportaban a la sociedad; posterior a las guerras que resultaban no solo en muertes, sino en alteraciones anatómicas y/o fisiológicas de los sobrevivientes, así como en la implementación de normatividad en el área de la seguridad social, hubo un cambio de paradigma, estableciéndose un modelo que propendía por la recuperación de dichas alteraciones (modelo médico o rehabilitador) en donde el personal de salud tenía un papel protagónico y paternalista, dejando de lado al individuo como sujeto de derechos; este último aspecto fue el principal cambio que trajo el modelo actual (modelos social), que a partir del Movimiento de Vida Independiente en la Universidad de Berkeley California, en 1960 buscó revindicar los derechos de las personas con discapacidad e inclusión social, más allá de la restauración de las condiciones físicas. 
En el marco de estos modelos la Organización Mundial de la Salud (OMS) desarrolló una serie de clasificaciones con el fin de definir y comprender mejor la discapacidad, siendo la primera la Clasificación Internacional de la Enfermedad (CIE) en 1893, que enlista diversos diagnósticos, los cuales siguen siendo de utilidad para la estandarización mundial de la información de diagnóstico en el ámbito de la salud, en su versión vigente CIE-11; sin embargo la CIE implica un paradigma biológico por lo que resultó necesario crear una nueva clasificación, es así como en 1970 surgió la Clasificación Internacional de la Deficiencia, la Discapacidad y la Minusvalía (CIDDM) que definió el primer término (deficiencia) como "anomalías de las estructuras corporales y de la función de las órganos o sistemas como consecuencias de la enfermedad", el segundo (discapacidad) como "consecuencias de las deficiencias desde el punto de vista del rendimiento funcional y de la actividad del individuo, con repercusión directa en la realización de las actividades que son consideradas normales" y el tercero (minusvalía) como "desventajas que experimenta el individuo como consecuencia de las deficiencias y discapacidades que afectan el desempeño que tiene la persona en la sociedad." La CIDDM continúo vigente en su segunda versión (CIDDM-2) hasta el año 2000 y un año después se publicó la Clasificación Internacional del Funcionamiento, de la Discapacidad y de la Salud (CIF) la cual proporciona un lenguaje común en torno a la salud y el funcionamiento, dando una perspectiva más social y positiva de la discapacidad (2).

La CIF, se estructura en dos partes (2), la primera incluye el funcionamiento $y$ discapacidad, que encierran las funciones y estructuras corporales, que son las funciones fisiológicas de los diferentes sistemas y las partes anatómicas del cuerpo respectivamente, así como las actividades (la realización de una tarea o acción por parte de un individuo sin dificultades) y la participación (acto de involucrarse en una situación vital). La segunda parte contiene los factores contextuales que constituyen el contexto completo de la vida de un individuo, que contempla factores ambientales que constituyen el ambiente físico, social y actitudinal en el que las personas viven y conducen sus vidas; y los factores personales que constituyen el trasfondo particular del individuo y su estilo de vida.

Es así como con base al modelo vigente y a la CIF, que será lanzada en su nueva versión en 2021, la discapacidad según la OMS incluye no solo deficiencias que son los problemas en las funciones o estructuras corporales, las limitaciones para el desempeño o la realización de actividades, sino también las restricciones en la participación, es decir los problemas que un individuo puede experimentar al involucrarse en situaciones vitales, para interactuar en su entorno social, así como los factores contextuales, expresados como facilitadores o barreras del medio ambiente o del propio individuo; por lo cual, al interactuar todos los elementos definidos anteriormente, la discapacidad implica una condición multifactorial (3). Por lo que un individuo puede tener alteraciones en sus órganos o sistemas a nivel anatómico o fisiológico a consecuencia de una enfermedad congénita, adquirida o un accidente, y continuar ejecutando las diferentes actividades y participando en sus diversos roles aunque con 
adaptaciones, o por el contrario tener limitación y restricción en estos, siendo determinante la interacción de los factores contextuales como el sistema de salud, la accesibilidad, la tecnología, las actitudes de la comunidad, así como la del propio individuo.

Por otra parte, pese a la conceptualización en torno a la discapacidad elaborada a lo largo de la historia, así como a los sistemas de clasificación de la OMS, se requiere de un arduo trabajo en la sociedad con el fin de promover, asegurar y proteger los derechos de las personas con dicha condición para favorecer su adecuado funcionamiento, inclusión y desarrollo personal, como lo son la Declaración Internacional de los derechos humanos y los tratados relacionados, las normas estipuladas la Organización Internacional del Trabajo (OIT) en 1983, la Convención Interamericana para la Eliminación de todas formas de discriminación contra las personas con discapacidad (Organización de los Estados Americanos (OEA) en 1999 y la Convención sobre los derechos de las personas con discapacidad en 2006 . $(4,5)$

Todo esto resulta importante, no solo para las personas en condición de discapacidad, sino para la sociedad en general, pues de acuerdo al informe mundial de discapacidad (4) en algún momento de la vida, gran parte de la población puede estar en riesgo de padecer algún tipo de discapacidad, ya que como se indicó anteriormente la discapacidad implica una alteración en la realización de las actividades y la restricción de la participación. Actualmente según el informe de la OMS y el Banco Mundial, en el mundo existen alrededor de 1.000 millones de personas con discapacidad, que corresponde a un $15 \%$ (2) y a nivel de América Latina y el Caribe de acuerdo al informe de discapacidad de las Américas se reporta una prevalencia del $12,6 \%$ la cual va en aumento debido a las enfermedades crónicas no trasmisibles, el cáncer, los trastornos mentales, el envejecimiento, el grado de accidentalidad y las enfermedades emergentes.

Este mismo documento resalta que las principales dificultades que afrontan las personas en situación de discapacidad están relacionadas con políticas, normas, financiación, accesibilidad y/o participación insuficientes, actitudes negativas y problemas con la prestación de servicios, por lo cual resulta indispensable realizar un diagnóstico de la situación en cada contexto para dar respuesta a las diferentes necesidades que reivindiquen el papel de las personas en condición de discapacidad en la sociedad y los entornos en los que estos se desenvuelven.

Por lo tanto la discapacidad va más allá de las alteraciones estructurales o funcionales, pues implica como se explicó la limitación en la realización de diversas actividades que incluye las actividades de la vida diaria, básicas cotidianas e instrumentales que permiten al individuo funcionar en diferentes niveles, redundan en independencia y a su vez favorecen la interacción con otros individuos en diversos ambientes, lo cual se expresa en la participación en diferentes roles, como lo son el familiar, social, laboral, recreativo y educativo, los cuales son importantes para el desarrollo de la persona en condición de discapacidad, pero también de la sociedad en general. 
Por lo general la discapacidad está asociada a una situación de desventaja y vulnerabilidad, esto debido al grado de dependencia de las personas a un tercero para la realización de las actividades, la dificultad de acceso a tecnologías de adaptación, así como a un escaso o nulo grado de participación, por lo que al reducir estas brechas no solo se favorece el desarrollo de las personas en condición de discapacidad sino al desarrollo social en general; en este sentido resulta importante resaltar la participación en el rol educativo, el cual ofrece un sinfín de oportunidades y posibilidades de desarrollo, ya que las cifras indican que solo entre el $10 \mathrm{al} 60 \%$ de personas en condición de discapacidad acceden a la básica primaria y entre el $15 \mathrm{al}$ $58 \%$ a la secundaria, en contraste aquellos que logran acceder a la educación primaria entre el 44 al 79\% la finalizan, mientras que la secundaria la finalizan entre el 4 al 63\%, cifras que varían respecto a cada país (6).

Con base en lo anterior, es de anotar que el nivel educativo se relaciona con el cumplimiento de derechos (Artículo 26 de la Declaración Universal de los Derechos Humanos, Artículo 24 de la Convención Internacional sobre los derechos de las personas con discapacidad) $(7,8)$, es rentable pues mejora los niveles de ingresos, la calidad de vida, contribuye a la eliminación de la discriminación y a la reducción de la vulnerabilidad, por lo que los Objetivos de Desarrollo Sostenible (ODS) en el objetivo número 4 resaltan la importancia de garantizar la educación de calidad inclusiva y equitativa, así como la promoción de oportunidades de aprendizaje permanente para todos, lo cual incluye por supuesto a las personas en condición de discapacidad, el trabajo conjunto y compromiso de los entes internacionales, nacionales, locales, instituciones educativas y en general a toda la sociedad.

En este contexto es preciso definir de manera adecuada el término "educación inclusiva" que es "un proceso permanente que reconoce, valora y responde de manera pertinente a la diversidad de características, intereses, posibilidades y expectativas de los niñas, niños, adolescentes, jóvenes y adultos, cuyo objetivo es promover su desarrollo, aprendizaje y participación, con pares de su misma edad, en un ambiente de aprendizaje común, sin discriminación o exclusión alguna, y que garantiza, en el marco de los derechos humanos, los apoyos y los ajustes razonables requeridos en su proceso educativo, a través de prácticas, políticas y culturas que eliminan las barreras existentes en el entorno educativo" (9), por tanto no es meramente integrar a la persona en condición de discapacidad en el salón de clases, sino promover su participación activa y el aprendizaje significativo acorde al marco conceptual y práctico de la CIF; para lo cual se han propuesto y construido lineamientos $y$ estrategias que orientan la puesta en marcha de la inclusión educativa que implica no solo la accesibilidad arquitectónica, sino la accesibilidad a la información, comunicación, tecnología, la reducción actitudes negativas, la exclusión y el empoderamiento de las personas en condición de discapacidad, que a su vez implica la satisfacción de todas sus necesidades y bienestar en general.

En este aspecto en 2019 en CaliColombia se celebró el vigésimo quinto aniversario de la "Declaración de UNESCO 
Salamanca: hacia la inclusión en la educación: estado, tendencias y desafíos" (10), en la cual se resaltó la importancia de la implementación de políticas de inclusión y equidad las cuales van de la mano, así como enfrentar los nuevos desafíos y la necesidad de investigación en torno a la discapacidad, inclusión y equidad, además se propusieron seis acciones para lograr una educación inclusiva y de equidad para el año 2030: 1) abordar de manera adecuada el concepto de inclusión (entendida como un proceso que permite superar las barreras que limitan el acceso y la participación en el rol educativo) y equidad (cada persona importa, e importa por igual), 2) identificar las barreras para la participación que incluye las de tipo físico como el acceso arquitectónico o tecnológico, social como las actitudes, psicológico como la autopercepción y a nivel de los sistemas y servicios como los costos o cupos, 3) fortalecer las habilidades docentes para promover la educación inclusiva, 4) diseñar currículos y sistemas de evaluación inclusivos lo cual en muchos casos requiere de ajustes razonables, 5) estructuración y administración de los sistemas educativos y 6) involucrar a la comunidad en general.

En el marco de la educación superior y en relación a lo mencionado anteriormente, en el I Seminario Regional sobre la inclusión de las personas con discapacidad en la Educación Superior en América Latina y el Caribe, organizado por el Instituto Internacional de la UNESCO para la Educación Superior en América Latina y el Caribe (IESALC) en 2005 (11) se resaltó que las instituciones de educación superior deben propender por una educación inclusiva que implica incluir este tema en los procesos misionales de las instituciones, así como en su visión y plan educativo, diseño, desarrollo y adaptación de programas incluyentes, la promoción de una cultura de diversidad, el fortalecimiento de la normatividad, mantener un registro de las personas en condición de discapacidad que incluye estudiantes, administrativos y docentes, capacitación permanente en torno a la inclusión, promover la accesibilidad en todo sentido, que incluye los sistemas de información, bibliotecas, acceso a la información y a los sistemas de evaluación, establecer fondos específicos de financiación para la inclusión educativa, crear, fortalecer y apoyar organizaciones que trabajen en pro del tema, así como las lideradas por las personas en condición de discapacidad, mantener procesos de información y sensibilización en la comunidad académica y la sociedad en general, documentar y difundir experiencias, así como implementar y fortalecer la investigación.

\section{REFLEXIÓN FINAL}

n conclusión, la discapacidad más


allá de la enfermedad, implica una perspectiva desde la función y el funcionamiento, así como desde el enfoque de derechos, resultando este concepto (discapacidad) de la compleja interacción entre todos estos aspectos, así como de los factores contextuales; por lo tanto si un individuo puede realizar diversas actividades aunque ello implique adaptaciones, que a su vez le permitan participar en los diferentes roles, entonces habremos reducido la brecha, que entre otras esferas el acceso a un educación de calidad que fomente el desarrollo personal y comunitario. 


\section{REFERENCIAS BIBLIOGRÁFICAS}

1. Velardez V. Los modelos de la discapacidad: un recorrido histórico. Revista empresa y humanismo. 2012;15(1):115-136.

2. Organización Mundial de la Salud. Organización Panamericana de la Salud. [Internet]. Clasificación Internacional del Funcionamiento, de la Discapacidad y la Salud CIF. 2001. [citado Junio 2020]. Disponible en: https://apps.who.int/iris/bitstream/handl e/10665/43360/9241545445_spa.pdf?seq uence $=1$.

3. Organización Mundial de la Salud [Internet]. Discapacidades. 2020. [citado julio 2020]. Disponible en: https://www.who.int/topics/disabilities/e/ 4. Organización Mundial de la Salud. Banco Mundial. [Internet]. Informe mundial sobre la discapacidad. 2011. [citado 30 noviembre 2020]. Disponible en: https://www.who.int/disabilities/world_r eport/2011/es/

5. Ministerio de Salud y Proyección Social. [Internet]. Política Pública Nacional de Discapacidad e Inclusión Social, Conpes Social. 2013. [citado Junio 2020]. Disponible en: https://www.minsalud.gov.co/proteccions ocial/promocionsocial/Discapacidad/Paginas/politicapublica.aspx

6. United Nations Educational, Scientific and Cultural Organization. [Internet]. Education and Disability. 2017. [citado 3 diciembre 2020]. Disponible en: https://unesdoc.unesco.org/ark:/48223/p f0000247516

7. Naciones Unidas. [Internet]. Declaración Universal de los derechos humanos. 1948. [citado 3 diciembre 2020]. Disponible en: https://www.un.org/es/universaldeclaration-human-rights/
8. Naciones Unidas. [Internet]. Convención sobre los derechos de las personas con discapacidad. 2006. [citado 3 diciembre 2020]. Disponible en: https://www.un.org/esa/socdev/enable/ documents/tccconvs.pdf

9. Ministerio de Educación. [Internet]. Decreto 1421 de 2017 por el cual se reglamenta en el marco de la educación inclusiva la atención educativa a la población con discapacidad. 2017. [Citado 3 diciembre 2020]. Disponible en: http://www.suinjuriscol.gov.co/viewDocument.asp?ruta=D ecretos $/ 30033428$

10. United Nations Educational, Scientific and Cultural Organization. [Internet]. Towards inclusion in education: status, trends and challenges: the UNESCO Salamanca Statement 25 years on. 2020. [citado 3 diciembre 2020]. Disponible en: https://unesdoc.unesco.org/ark:/48223/p f0000374246?fbclid=IwAR2BDzzOWHH6 NQb0KOcxjTqS1aso61zVI3EPhlGNcfaaGWhsm2ZlqMT_XU

11. Organización de las Naciones Unidas para la Educación, la Ciencia y la Cultura. [Internet]. I Seminario Regional sobre la inclusión de las personas con discapacidad en la Educación Superior en América Latina y el Caribe. [Internet]. Caracas; 2005 [citado 1 diciembre 2020]. Disponible en: http://www.debatecultural.net.ve/Observ atorio/UnescoSeminarioRegional.htm

Conflicto de Intereses: Los autores declaran que no existe conflicto de intereses para la publicación del presente artículo científico.

Financiamiento Los autores declaran no haber recibido financiamiento

Agradecimientos: Los autores agradecen a las personas con discapacidad y de todos quienes se esfuerzan por la inclusión; personal de salud, personal de educación, investigadores y la sociedad en general. 